phosphamide, and melphalan given individually produce a remission in a quarter to a third of patients with advanced ovarian tumours, but combinations of these drugs have been disappointing, with no increase in that proportion. The addition of cis-platinum diamminodichloride even in relatively low doses has doubled these response rates. ${ }^{16}$ Other agents, such as hexamethyl melamine, adriamycin, ifosfamide, peptichemio, and the new podophyllotoxins (including VP16), are being evaluated. Even the best chemotherapeutic combinations, however, may be ineffective without surgical treatment to reduce the bulk of the tumour.

Current management of advanced ovarian carcinoma requires the removal of as much tumour as safely possible at initial laparotomy, along with both ovaries, uterus, and omentum (in which sites occult disease may reside). In order to render any inoperable disease removable, a chemotherapeutic response should be established as soon as possible, and, after maximum response, another laparotomy should be performed to remove any residual tumour, along with multiple biopsies of suspicious areas. Further adequate chemotherapy is indicated to eradicate any residual macroscopic or microscopic disease. Greater accuracy in the choice of chemotherapy for individual patients may, in the future, be obtained by drug selection challenges on the xenografted tumours in immune deficient mice or cell colonies obtained from laparotomy specimens.

Advanced ovarian carcinoma is found in as many as $70 \%$ of all patients at presentation. The median survival is about eight months from diagnosis. Any improvement on that grim prognosis requires a combination of surgical expertise and determined chemotherapy; and compromise in either modality is likely to result in relapse and rapid death.

1 Haenszel, W, and Kurihara, M, Fournal of the National Cancer Institute, $1968,40,43$.

2 Toth, B, Cancer Research, 1970, 30, 2583.

${ }^{3}$ British Medical fournal, 1972, 2, 365.

4 Schoenberg, B S, Greenberg, R A, and Eisenberg, H, Fournal of the National Cancer Institute, 1969, 43, 15.

5 Wynder, E L, Dodo, H, and Barber, H R K, Cancer, 1969, 23, 352.

6 Sterwart, H L, et al, fournal of the National Cancer Institute, 1966, 37, 1.

7 Jussawalla, D J, et al, British Fournal of Cancer, 1970, 24, 56. 8 Christian, C D, American fournal of Obstetrics and Gynecology, 1971, 111,
529 .

${ }^{9}$ Berlin, N I, et al, Annals of Internal Medicine, 1966, 64, 403.

10 Committee on Safety of Medicines, Carcinogenicity Tests of Oral Contraceptives. London, HMSO, 1972.

${ }^{11}$ Adelstein, A M and Donovan, J W, British Medical fournal, 1972, 4, 629.

12 West, R O, Cancer, 1966, 19, 1001.

13 Osborne, R H, and Degeorge, F V, American Fournal of Human Genetics. $1963,15,380$.

14 Clemmesen, J, Fuglsang-Frederiksen, V, and Plum, C M, Lancet, 1974, 1, 705 .

15 Rosenberg, B, et al, Nature, 1969, 222, 385

16 Wiltshaw, E, and Barker, G $\mathrm{H}$, to be published.

\section{Transplant Olympics}

Last month 200 men and women between the ages of 14 and 44 assembled at Castle Field in Portsmouth to take part in the second Transplant Olympics. As athletes they shared one characteristic: each had a well-functioning kidney transplant. Their performance in the 33 separate events may not have reached Olympic or even sports clubs standards, but their times were certainly better than might be expected from, say, their doctors. The 1500 metres was won in a modest six minutes by Paul West of Liverpool-the sort of time that any ordinary young man or woman in the street might be able to accomplish with some training. Again, the tennis champions, men and women, were certainly not of Wimbledon class but they would have felt comfortable on the first ladder of their local club.

These games were not, then, for the handicapped: they were for men and women with transplant kidneys, of one to eight years' vintage, who wanted to demonstrate their normality. Competitors (six for each team) came from England, Scotland, Wales, and Northern Ireland and from countries overseas as far afield as Mexico, Israel, and Greece. Their aim was to be ambassadors for kidney transplantation and to draw attention to their normality in the hope that this would persuade the spectators to become potential kidney donors and thus help the thousands of patients on dialysis to achieve a similarly normal, independent state of existence. Over 5000 donor cards were signed at the meeting and taken away.

The organising committee and its chairman, transplant surgeon Mr Maurice Slapak of St Mary's Hospital, Portsmouth, decided that no individual records of times or distances would be kept, though gold, silver, and bronze medals were awarded to the winners of the varying events. The champion team was Eire, with its flying Irishman Maxie Scully, the winner of three gold medals in highly creditable times. The most colourful entrant was a Mexican who did not compete-he had a wooden leg-but instead he entertained the others with his guitar.

Six separate bids were entered by countries who wanted to stage next year's Olympics, including an imaginative Canadian bid for a Winter Olympics in 1981 in the Rockies. The International event was awarded to the United States and will be staged in September 1980 at Lake Placid. The Transplant Olympic Games to choose the British team will be staged in August 1980 in Birmingham.

\section{Liver dysfunction in inflammatory bowel disease}

Patients with ulcerative colitis or Crohn's disease may develop extraintestinal complications of the skin, eyes, joints, or renal tract. ${ }^{1}$ Various hepatobiliary disorders are also associated with inflammatory bowel disease, including pericholangitis, ${ }^{2}$ fatty change, chronic active hepatitis, cirrhosis, granulomas, amyloidosis, hepatic abscesses, ${ }^{3}$ gall stones, ${ }^{45}$ sclerosing cholangitis, ${ }^{6}$ and carcinoma of the biliary tract. ${ }^{7} 8$

Minor histological abnormalities are equally common in Crohn's disease and ulcerative colitis, but their prevalence depends on the source of the pathological material. Roughly two-fifths of surgical biopsy and postmortem specimens show fatty change, a reflection of the poor nutritional state or septicaemia of some patients during an acute attack. ${ }^{910}$ Pericholangitis, also called portal triaditis, is recognised histologically by cellular infiltration of the portal tracts, portal fibrosis, and concentric fibrosis around the bile ducts, and is found in over half of surgical biopsy specimens. ${ }^{910}$ In clinical studies most liver biopsy samples have been obtained from patients with abnormal serum enzyme concentrations or bromsulphalein retention, and in one such series fatty change and pericholangitis were each found in about $7 \%$ of patients with inflammatory bowel disease. ${ }^{11} 12$ It is important not to mistake such histological changes, which are of little clinical importance, for those of serious liver disease. Fortunately the latter is 
uncommon: cirrhosis develops in fewer than $3 \%$ of patients with inflammatory bowel disease. ${ }^{111} 12$ Chronic active hepatitis, amyloidosis, granulomas, and hepatic abscesses are also rare, and taken together occurred in only $1-2 \%$ of patients in several large series. ${ }^{1}$ 9-12

These reassuring figures have been confirmed in a recent survey from Birmingham of over 1200 patients with inflammatory bowel disease. ${ }^{13}$ Abnormal liver function tests were present at some time in just over $8 \%$ of patients, but in many cases they developed transiently after surgery and were associated with abdominal sepsis. One-fifth of patients with abnormal liver function tests had pericholangitis. Cirrhosis was found in three of the 517 patients with Crohn's disease and in 11 of the 720 cases of ulcerative colitis, giving an overall prevalence of about $1 \%$.

The cause of chronic liver disease in these patients remains obscure. It is not related to blood transfusion, drug treatment, or other systemic complications. ${ }^{9}$ Portal bacteraemia has been suggested as a possible mechanism, but cultures of liver biopsy specimens and portal blood samples are usually sterile. ${ }^{40-12}$ Moreover, pericholangitis progresses to cirrhosis only rarely. The effect of colectomy is uncertain, for, although liver histology may improve after resection, ${ }^{14}$ differences in biopsy technique, sampling error, and possible observer bias make this observation difficult to interpret-and we have to remember that patients with cirrhosis may enjoy good health for many years after colectomy. Although resection may retard or reverse the progression of the liver disease (or delay the onset of hepatic encephalopathy), possibly also cirrhosis associated with inflammatory bowel disease runs a particularly slow course or laparotomy leads to the detection of liver disease at a very early stage.

Gall stones are present in up to a third of patients with Crohn's disease of the terminal ileum-at least three times the prevalence in control subjects. ${ }^{4}$ Ileal disease or resection results in the malabsorption of bile salts with reduction in their pool and supersaturation of the bile with cholesterol. ${ }^{15}$ The tenfold increase in these patients in the incidence of carcinoma of the bile ducts and gall bladder is more difficult to explain. The Birmingham workers found such neoplasms in eight of their patients with ulcerative colitis. They differ from the usual carcinomas of the biliary tract by developing in younger patients without gall stones, and one puzzling feature is that they may develop many years after colectomy. ${ }^{\text {? }}$ Sclerosing cholangitis may affect the intrahepatic or extrahepatic biliary tree, with diffuse narrowing of the bile ducts or multiple strictures separated by saccular dilatation, ${ }^{6}$ and is sometimes associated with finding pericholangitis in the liver biopsy sample. ${ }^{16}$ Sclerosing cholangitis was diagnosed in seven $(1 \%)$ of patients with ulcerative colitis in the Birmingham series.

How can we recognise these disorders? Although liver function tests may be normal in patients with fatty liver, pericholangitis, or even cirrhosis, measurement of serum enzyme concentrations such as alkaline phosphatase remains the best screening test generally available. Serious liver disease is more common in patients with extensive disease of the colon and a reasonable plan would be to check liver function tests every 12 months in such patients. A rise in serum enzyme concentrations or the development of pain in the right upper quadrant of the abdomen, enlargement of the liver, jaundice, or pruritus would all indicate the need for further investigation; and if the patient has had a colectomy (no matter how many years previously) the possibility of a bile duct carcinoma should be considered. Although liver biopsy is safe in patients with obstruction of the large ducts, ${ }^{17}$ radiographic assessment of the biliary tree will be more appropriate in most cases. Intravenous cholangiography may be unsuccessful in patients with jaundice, when ultrasonography may be a helpful non-invasive test to determine whether or not the bile ducts are dilated. Percutaneous transhepatic cholangiography will outline the biliary tree in over $90 \%$ of patients with dilated ducts, but endoscopic retrograde cholangiography is the more useful procedure in those with non-dilated ducts. ${ }^{18}$

The radiographs may show gall stones or a single neoplastic stricture, either of which may be treated surgically. The management of sclerosing cholangitis is less satisfactory. Steroids, azathioprine, and antibiotics have all been used but are of uncertain benefit. ${ }^{619}$ Dilatation of the extrahepatic bile ducts with insertion of a $\mathrm{T}$-tube for several months may allow inflammation to resolve without the formation of new strictures. ${ }^{20}$ Nevertheless, the outlook for most patients with sclerosing cholangitis is poor: they may develop ascending bacterial infection, progressive jaundice, intractable pruritus, and secondary biliary cirrhosis.

\footnotetext{
${ }^{1}$ Greenstein, A J, Janowitz, H D, and Sachar, D B, Medicine (Baltimore), 1976, 55, 401 .

${ }^{2}$ Mistilis, S P, Annals of Internal Medicine, 1965, 63, 1.

3 Watts, H D, American fournal of Digestive Diseases, 1978, 23, $41 \mathrm{~s}$

4 Cohen, S, et al, Gastroenterology, 1971, 60, 237.

5 Baker, A L, et al, American fournal of Digestive Diseases, 1974, 19, 109.

${ }^{6}$ British Medical fournal, 1976, 2, 1090.

${ }^{7}$ Ritchie, J K, et al, Quarterly fournal of Medicine, 1974, 43, 263.

${ }^{8}$ Akwari, O E, et al, Annals of Surgery, 1975, 181, 303.

9 Eade, M N, Annals of Internal Medicine, 1970, 72, 475.

${ }_{10}$ Eade, M N, et al, Annals of Internal Medicine, 1971, 74, 518.

11 Perrett, A D, et al, Quarterly fournal of Medicine, 1971, 40, 211

12 Perrett, A D, et al, Quarterly fournal of Medicine, 1971, 40, 187.

${ }^{13}$ Dew, M J, Thompson, H, and Allan, R N, Quarterly fournal of Medicine, $1979,48,113$

${ }^{14}$ Eade, M N, Cooke, W T, and Brooke, B N, Annals of Internal Medicine, 1970, 72, 489

${ }^{15}$ Marks, J W, et al, American fournal of Digestive Diseases, 1977, 22, 1097

${ }^{16}$ Blackstone, M O, and Nemchausky, B A, American Fournal of Digestive Diseases, 1978, 23, 579.

17 Morris, J S, et al, Gastroenterology, 1975, 68, 750

18 Elias, E, et al, Gastroenterology, 1976, 71, 439.

19 Mistilis, S P, Skyring, A P, and Goulston, S J M, Australasian Annals of Medicine, 1965, 14, 286.

20 Schwartz, S I, The Surgical Clinics of North America, 1973, 53, 1161.
}

\section{Wanted: a new wound dressing}

With the notable exception of the burn, for which the late A B Wallace successfully reintroduced open treatment in the late 1940 s, our natural inclination is to cover any wound. Paré's over-quoted statement "I dressed him; God healed him" attests to the orthodoxy of a practice that goes back at least to the Smith papyrus. Though in each generation some surgeons expose the sutured wound to the air, with or without some ritualistic practice such as a spray of fast-drying plastic, they are the exception. Perhaps dressings are more social than surgical, on the one hand preserving clothes and bed linen from blood and exudate and on the other hand shielding the patient from the affront to his body's integrity.

If wounds are to be dressed then let it be with the most efficient materials. T D Turner ${ }^{1}$ at the Surgical Dressing Research Unit of the Welsh School of Pharmacy has recently looked at the types of dressing required and the commonly available products ostensibly designed to meet the clinical need. He distinguishes arbitrarily but conveniently between dressings 